Revue d'histoire de l'enfance « irrégulière »

Le Temps de l'histoire

Hors-série | 2001

Histoire et justice, panorama de la recherche

Le parricide à l'aune de la justice au XIXème siècle

Sylvie Lapalus

(2) OpenEdition

Journals

Édition électronique

URL : http://journals.openedition.org/rhei/440

DOI : 10.4000/rhei.440

ISBN : 978-2-7535-1641-0

ISSN : 1777-540X

Éditeur

Presses universitaires de Rennes

Édition imprimée

Date de publication : 15 novembre 2001

Pagination : 141-154

ISSN : 1287-2431

Référence électronique

Sylvie Lapalus, " Le parricide à l'aune de la justice au XIXème siècle », Revue d'histoire de l'enfance " irrégulière » [En ligne], Hors-série | 2001, mis en ligne le 31 mai 2007, consulté le 19 avril 2019. URL http://journals.openedition.org/rhei/440 ; DOI : 10.4000/rhei.440 


\section{Le parricide à l'aune de la justice au XIXème siècle}

A l'échelle de la France, avec une moyenne annuelle de douze affaires jugées aux assises, le parricide est au XIXème siècle un crime d'exception. Cette relative rareté est toutefois sans commune mesure avec la charge symbolique que revêt le meurtre du père dans une société qui fait de la famille patriarcale une valeur cardinale et voit dans le régicide la transposition politique du parricide : qu'on pense aux incidences de l'affaire Fieschi, en 1836, sur le sort de Pierre Rivière, le parricide normand sorti de l'oubli par Michel Foucault et son équipe. ${ }^{(1)}$

Tout au long du XIXème siècle, la définition légale demeure identique : l'article 299 du Code pénal de 1810 fait du parricide, qui emporte la peine de mort, le meurtre des père et mère légitimes, naturels ou adoptifs, ou de tout autre ascendant légitime. En droit, le parricide constitue donc l'un de ces rares crimes pour lesquels c'est d'abord la qualité de la victime qui, quelle que soit l'arme utilisée, détermine juridiquement la catégorie pénale. Placé au sommet de la hiérarchie pénale, le parricide est aux termes de l'article 323 du Code un acte sans excuse, l'enfant devant tout souffrir plutôt que porter une main sacrilège sur ses parents. Tel un père, le législateur favorise la protection des ascendants contre leurs descendants alors que, dissymétrie flagrante de la législation pénale, le crime inverse, celui que peut commettre un parent sur son enfant, est au moins jusqu'en 1898 réprimé selon les règles du droit commun.(2)

Pour étudier la manière dont la justice se saisit du parricide au XIXème siècle, il semble nécessaire de considérer successivement le crime puis le criminel : à l'image de la société au nom de laquelle elle agit, la justice a beaucoup de peine à appréhender l'acte criminel en tant que tel, à la fois dans sa réalité matérielle et plus manifestement encore dans sa

\section{Sylvie Lapalus}

(1) Michel FOU-

CAULT [dir.], Moi,

Pierre Rivière, ayant égorgé ma mère, ma saur et mon frère... Un cas de parricide au XIXème siècle, Paris, Gallimard/Julliard, 1973, $350 \mathrm{p}$.

(2) Voir dans le Temps de l'bistoire $\left(\mathrm{n}^{\circ} 2\right)$ les actes du colloque "Cent ans de répression des violences à enfants”, (CNFE-PJJ/Paris I), qui s'est tenu le 27 avr. 1998.

Sylvie Lapalus / pp. 141 à 154 
(3) Cette étude s'appuie essentiellement sur un corpus de dossiers judiciaires de 771 affaires de parricide jugées en France entre 1825 et 1913 ainsi que sur les données statistiques fournies par le Compte général de l'administration de la justice criminelle en France qui paraît à un rythme annuel à partir de 1827 (volume couvrant l'année 1825). Sur la richesse et les limites de cette source, la référence de base est : Michelle PERROT et Philippe ROBERT [prés.], Compte général de l'administration de la justice criminelle en France pendant l'année 1880 et rapport relatif aux années 1826 à 1880, reprint, Genève/Paris, Slatkine Reprints, 1989, 30 CLXXII p.

(4) Sylvie LAPALUS, "Le parricide comme exacerbation des violences familiales", Trames, n 5 , juin 1999 , dimension juridique et transgressive ; aussi réinvestit-elle progressivement le parricide au prix d'un changement de perspective en faisant glisser son regard du crime, difficile à envisager, vers le criminel, a priori plus facile à jauger. Pour n'être pas spécifique au parricide puisqu'il renvoie à une attitude générale au XIXème siècle, ce déplacement du regard judiciaire n'en est pourtant que mieux perceptible à l'occasion de ce genre d'affaire dans la mesure où il permet de penser un acte par nature impensable, un acte qui en mettant le phénomène criminel à nu, ouvre un abîme de mystère pour les contemporains. ${ }^{(3)}$

\section{Un crime difficile à appréhender}

Au XIXème siècle, si la justice éprouve de sérieuses difficultés à appréhender le parricide, c'est à la fois pour des raisons qui sont inhérentes au crime et pour d'autres plus propres au fonctionnement pénal et judiciaire mais qui toutes ressortissent en dernière analyse à la signification du parricide et à ses enjeux profonds. ${ }^{(4)}$

\section{Les obstacles à la saisie judiciaire}

Parce qu'un tel drame se noue dans le huis clos familial, plus de $32 \%$ des parricides ${ }^{(5)}$ sont portés tardivement à la connaissance de la justice et plus d'un quart des cas finalement jugés ${ }^{(6)}$ ont posé à un moment ou à un autre de la procédure le problème de la nature criminelle du décès, que ce dernier ait été camouflé préalablement en mort naturelle, en accident ou maquillé en suicide.

Crime majoritairement rural, le parricide confronte régulièrement la justice à l'opacité du cercle familial, confortée au XIXème siècle par le relatif resserrement du noyau domestique. C'est non sans mal que les autorités judiciaires pénètrent dans l'intimité familiale même dans le cas d'un crime aussi grave que le parricide qui soude au contraire plus que jamais la communauté des proches soucieuse avant tout de préserver son capital d'honneur et d'éviter que l'infamie ne soit portée sur la place publique. Afin de dissimuler l'homicide, les libertés prises avec les pratiques funéraires et surtout la législation sur les inhumations ${ }^{(7)}$ ne sont pas rares : on fait en sorte d'écarter les voisins bien intentionnés venus aider 
à la toilette funéraire ou bien on leur présente un cadavre dont toute marque de violence a été effacée ; on n'hésite pas à choisir des ensevelisseuses de confiance ou alors celles d'un village voisin pour couper court à toute rumeur gênante ${ }^{(8)}$; on s'arrange pour hâter l'inhumation avant les 24 heures réglementaires ${ }^{(9)}$ et empêcher ainsi la visite redoutée de l'officier de l'état civil sous prétexte, par exemple, que le cadavre exhalerait une odeur pestilentielle. ${ }^{(10)}$ Les familiers n'ont pas toujours l'autorité morale ni même tout simplement le désir de s'immiscer dans des affaires privées à un moment où de surcroît les sociabilités traditionnelles commencent à connaître un relatif déclin. En 1853, en acquittant contre l'avis du président des assises un accusé qui comparaît pour tentative de parricide, les jurés de la Vienne entérinent cette maxime populaire selon laquelle il faut laisser aux membres de la famille le soin de «laver [entre eux] leur linge sale ${ }^{(11)}$ ». La force du lien familial ne facilite guère la quête de vérité entreprise par la justice et les preuves morales ou exclusives seul l'accusé ayant pu commettre un tel acte en l'absence d'ennemis connus - ne peuvent pallier le manque de preuves matérielles.

La mise en évidence du corps du délit par la justice est de ce fait rendue particulièrement ardue. Les procédures d'investigation auxquelles se trouvent soumises les autorités judiciaires restent encore incertaines au XIXème siècle : dans le cadre familier du foyer domestique ou d'un espace connu, il n'est pas rare qu'un inculpé parvienne à cacher des pièces à conviction lors de l'état des lieux ${ }^{(12)}$ ou qu'un membre de la famille se charge discrètement d'éliminer les traces du crime. ${ }^{(13)}$ Accusée d'avoir fait disparaître un mouchoir constituant une preuve à charge contre son fils, la dame Baloche répond avec vigueur que "ce n'est pas à une mère à accuser son enfant ${ }^{(14)}$ ". En outre, la fragilité de certains diagnostics médicaux, surtout quand il sont éventuellement posés à la va-vite par des médecins de campagne, brouille la visibilité du crime. Par leur force de conviction, sinon pour la pression qu'ils sont susceptibles d'exercer, les proches agissent aussi sur l'officier de santé qui peut être conduit plus ou moins habilement à négliger les signes de l'homicide. Insidieusement aidé dans son diagnostic, le médecin parfois peu formé, voire peu consciencieux ou pp. 138-139.

(5) Soit, au sein de notre corpus, 70 sur 216 affaires pour lesquelles nous disposons de ces informations.

(6) Soit 166 cas sur 649 .

(7) Code civil, art. 77.

(8) Comme dans

l'affaire Denonvilliers où la fille et la mère, toutes deux complices du meurtre commis sur leur père et mari, Nicolas Denonvilliers, font appel aux services d'une ensevelisseuse d'un autre village prétendument moins chère, à qui elles demandent d'accomplir l'opération le plus vite possible et de «commencer par la tête " afin de cacher les marques de pression encore visibles sur le cou du cadavre (Archives nationales (AN), BB/24/2002.

DOS. 2704/S7,

Florentine DENONVILLIERS et CARLE, épouse DENONVILLIERS, 1827). 
(9) Le jour même de la mort de son grand-père Pierre-Louis Village, Eugène Nicolas, le meurtrier, s'était rendu chez le fossoyeur et le sonneur de cloches en leur ordonnant de presser le glas, la confection de la bière et les funérailles alléguant que " cela pressait ", "que si Village n'était pas enterré dans la journée, cela ne l'arrangerait pas » (AN, BB/20/208/1, aff. NICOLAS, 1858).

(10) Gazette des tribunaux, 27 nov. 1835, aff. RIZOU.

(11) AN, BB/20/168/1, aff. POISSARD, Vienne, 1853.

(12) En 1896, par exemple, pendant que le maire a le dos tourné, François Hocquel, coupable du meurtre de son père, fait disparaître un bourroir à cartouches compromettant (Gazette des tribunaux, 21 juin 1896, aff. HOCQUEL).

(13) Dans l'affaire plus rarement complaisant, en arrive par exemple à reconnaître les symptômes du choléra chez un vieillard en réalité empoisonné par ses enfants ${ }^{(15)}$ ou attribue tout simplement à une congestion cérébrale consécutive à un abus d'alcool le trépas d'une mère causé par un violent coup assené sur le crâne ! ${ }^{(16)}$ En 1863 dans les Landes, malgré la présence pourtant évidente d'ecchymoses et d'empreintes de mains sur le cadavre, des erreurs ont manifestement été commises lors de l'établissement du décès du père Colobie : la mort s'avère finalement être le fait de la strangulation, et non de l'asphyxie par suspension volontaire ainsi que l'avait d'abord déclaré le médecin expert. A propos de cette affaire, tout en se faisant l'écho du président des assises des Hautes-Pyrénées mécontent de "l'imprécision des hommes de l'art » et appelant de ses vœux l'institution dans chaque ressort de médecins légistes capables de répondre aux besoins des informations et expertises criminelles, ${ }^{(176)}$ la direction des Affaires criminelles et des Grâces rappelle à l'ordre le conseiller à la cour impériale de Pau :

"Cette question mériterait un examen sérieux, mais en attendant qu'elle soit résolue, il y a des instructions de la Chancellerie, fréquemment renouvelées, qui recommandent aux procureurs impériaux d'indiquer à l'avance à leurs auxiliaires les hommes de l'art qu'il convient d'employer de préférence, comme méritant la confiance des tribunaux et il semble que ces circonstances soient négligées dans votre ressort. ${ }^{(18)} »$

Aussi les instances judiciaires en sont-elles réduites à reconnaître sans ambages leur dette à l'égard des bruits accusateurs auxquels elles s'empressent de recourir dès que l'occasion se présente : la "rumeur" ${ }^{19)}$ signale le crime aux autorités publiques ou leur désigne les coupables dans plus de $28 \%$ des affaires de parricide. ${ }^{(20)}$ A Dourdan, près de Rambouillet, c'est la rengaine fredonnée par les enfants du lieu qui, en 1839, met finalement la justice sur la piste d'un matricide commis quelques années plus tôt : "Fin, fin, il a assassiné sa mère pour avoir son bien! (21)" Mais aux difficultés de la saisine judiciaire viennent encore s'ajouter les problèmes soulevés par la définition légale du crime. 


\section{Une définition du crime problématique}

Quoique constante au XIXème siècle et précise dans sa formulation, la définition juridique du crime de parricide ne va pas sans susciter de vives polémiques chez les pénalistes et poser plus encore de graves problèmes aux magistrats chargés de la mettre en application, le Code pénal faisant en effet surgir deux controverses.

La première concerne la nature juridique précise du crime de parricide. A partir du milieu des années 1830, sous l'emprise de la réforme de certaines dispositions du Code d'instruction criminelle, ${ }^{(22)}$ apparait une divergence d'interprétation entre les magistrats siégeant en cour d'assises qui, fidèles en cela à la doctrine, font du parricide un meurtre aggravé et, la Cour de cassation qui persiste à voir dans le parricide un crime suigeneris, c'est-à-dire un crime spécial distinct des autres homicides par la spécificité de sa définition et de sa peine. ${ }^{(23)}$ La controverse est d'importance puisqu'elle influe sur la formulation des questions posées aux jurés : en cas de meurtre aggravé, ceux-ci doivent répondre à deux questions l'une sur le fait du meurtre, l'autre sur la filiation ; en revanche, s'agissant d'un crime sui generis, le jury n'a plus à répondre qu'à une seule question qui englobe les différentes circonstances du crime. Outre des considérations procédurales, ${ }^{(24)}$ cette divergence d'interprétation traduit bien une appréhension différenciée du crime. La Cour de cassation défend une approche globale et abstraite du crime : le parricide, crime suprême dans la hiérarchie pénale, ne saurait être envisagé que de manière monolithique, comme un tout ne pouvant se décomposer. De leur côté, les magistrats des cours d'assises se sentent tenus dans leur pratique d'aborder le crime de manière analytique en en dissociant les différents éléments afin d'éviter de possibles "acquittements scandaleux" selon la formule consacrée au XIXème siècle. En cas de question unique et surtout de doute sur la filiation de l'accusé, les jurés, effrayés par la responsabilité qui leur incombe quant au sort de l'accusé, pourraient en effet être tentés de rejeter la question tout entière alors que la dissociation des différentes composantes du crime rend la réponse sur le meurtre indépendante de celle sur la filiation.
Bazin, invoquant l'usage

du pays rémois selon

lequel la maison d'un

mort doit être nettoyée, les proches de la famille détruisent tous les indices gênants qui se trouvent sur les lieux du crime (AN, BB/20/132, aff. BAZIN, Marne, 1845).

(14) Archives départementales (AD) de Seineet-Oise, 2 U 367, aff. BALOCHE, 1857, audition de la mère de l'accusé datée du 2 nov. 1851.

(15) AD Doubs, U 4594, aff. GAULARD, 1874.

(16) AN, BB/24/2039/

1. DOS. 1132/S73, Augustin GUYARD, 1873.

(17) Sur ce point, consulter Frédéric CHAUVAUD, "Râles des moribonds et cris post-mortem au XIXème siècle : la déposition des victimes de mort violente", in Actes du colloque du CEH de l'université de Bourgogne sur 
"Les victimes de

l'Antiquité à l'époque contemporaine" (Dijon, 7-8 oct. 1999), sous presse ; voir également du même auteur Les Experts du crime. La médecine légale en France au XIXème siècle, Paris, Aubier, 2001, 304 p.

(18) AN, BB/20/255, aff. COLOBIE, Landes, 1863.

(19) Les magistrats utilisent indifféremment les termes de "clameur", "bruit", "opinion”, "conscience publique", "voix populaire".

(20) Soit 90 affaires sur 318 à l'intérieur de notre corpus.

(21) AD Seine-et-Oise, 2U 269, aff. BRÉANT, 1839.

(22) Lois du 9 sept. 1835 et du 13 mai 1836.

(23) Cf. infra.

(24) La Chambre criminelle de la Cour de cassation prétend de la sorte créer au profit de l'accusé une garantie supplémentaire qui lui
La deuxième controverse suscitée par le Code pénal concerne l'assimilation entre le fils parricide et son complice, assimilation dénoncée avec constance par plus d'un juriste au cours du XIXème siècle. En vertu de l'article 59 du Code, le complice d'un fils parricide est en effet assimilé au parricide lui-même quand bien même il n'aurait aucun lien de parenté avec la victime, tandis qu'un fils qui provoque un étranger au meurtre de son propre père ou qui s'y associe indirectement comme complice, n'est pas reconnu coupable de parricide mais de meurtre simple. En 1827, lors de l'affaire Dupré au cours de laquelle sont mis en accusation une fille et son époux, les propos de l'avocat de la défense sont sans équivoque :

"Le fils, meurtrier de son père, ne peut pas infuser dans les veines de son complice le sang qui coule dans les siennes; il ne peut pas lui rendre communs les rapports de nature qui existent entre lui et sa victime. L'un sera parricide; mais l'autre se sera jamais qu'un meurtrier. Dira-t-on que le fait de complicité dans un parricide offre une aggravation de criminalité, qui ne permet pas de le confondre avec celui de complicité dans un meurtre ordinaire? Cela est vrai ; mais qu'est-ce que cette nuance morale, auprès de cette révolte du sang, qui seule motive et légitime la peine du parricide? Non, la loi civile, quelque absolue que soit l'assimilation qu'elle fait du complice et de l'auteur principal, n'a pu ni voulu violenter la loi de nature et étendre le parricide hors des relations de paternité et de filiation. ${ }^{(25)}$ "

Bien qu'inchangée, la définition du parricide est donc loin de faire l'unanimité. Mais plus encore que la définition juridique du crime, c'est bien la qualification de l'acte criminel qui pose problème.

\section{Une qualification malaisée de l'acte criminel}

$\mathrm{Au}$ XIXème siècle, le parricide plonge de toute évidence le corps judiciaire dans le désarroi.

Les magistrats envisagent non sans réticence la possibilité d'un crime qui fait si unanimement horreur : le parricide surgit comme un contresens absolu dans une société dont il va jusqu'à saper les fondements. Malgré leurs exigences répressives, les présidents des assises sont égale- 
ment contraints, on l'a vu, de composer avec la réaction des jurés angoissés à l'idée d'endosser la responsabilité d'un verdict irrémédiable. C'est aussi sans compter que l'attitude des victimes, quand elles survivent ne serait-ce qu'un instant, apparaît des plus ambivalentes : malgré la gravité de la violence subie, $45 \%$ des parents ${ }^{(26)}$ choisissent en définitive de disculper leur agresseur de manière plus ou moins franche et délibérée. Face à des affaires dont les suites judiciaires s'avèrent somme toute aléatoires, les représentants de la justice préferent se montrer prudents.

C'est pourquoi la qualification de l'acte criminel se révèle relativement confuse et que les frontières entre les incriminations apparaissent mouvantes, souvent au détriment des victimes dont les blessures, au mépris des évidences, en arrivent non seulement à être sous-estimées ou occultées mais même totalement niées. Souvent pressés par les défenseurs, les présidents des assises ont ainsi tendance à délaisser l'accusation de parricide proprement dite au profit de celle, moins grave, de " coups ayant entraîné la mort sans intention de la donner (27) ", incrimination introduite en 1832. De même, la frontière apparaît tout aussi fluctuante entre la tentative de parricide, théoriquement assimilable au parricide lui-même - bien que ce soit loin d'être toujours le cas dans la réalité - et les " coups et blessures à ascendants ${ }^{(28)}$ " évidemment moins sévèrement sanctionnés. Et, quoique juristes et magistrats s'accordent communément à juger cette pratique impossible dans le cas d'un crime aussi grave, ${ }^{(29)}$ le parricide peut aussi donner lieu à une forme indirecte de correctionnalisation : ainsi en 1865, les moyens de défense invoqués par l'avocat d'Antoine Séverac pour obtenir l'acquittement de son client, suspect de parricide, permettent de déférer ensuite ce dernier à la juridiction correctionnelle pour homicide par imprudence. ${ }^{(30)} \mathrm{Si}$ certains magistrats ne manquent pas de voir dans ce procédé une transaction contraire à la vérité et même "un expédient peu digne de la justice (31) ", d'autres n'ont pas les mêmes scrupules, tel ce président de la cour d'assises des Vosges qui, en 1858, usant là d'arguments classiques pour justifier le principe de correctionnalisation, estime pour sa part qu'il est " du devoir des magistrats de réduire l'accusation à ses plus minimes proportions afin d'obtenir plus sûrement une répression ${ }^{(32) » .}$ serait refusée si la filia-

tion était placée au rang de circonstance aggra-

vante : la loi de 1835

impose en effet au jury

d'indiquer le nombre de voix obtenu en réponse à la question principale, mais non pour les circonstances, fussent-elles aggravantes. Dans le cas d'un verdict de culpabilité prononcé par les jurés à la majorité simple, la Cour peut, à la majorité de ses voix, annuler ce verdict et renvoyer l'affaire à une session ultérieure si elle estime que le jury s'est trompé sur le fond ; en cas de majorité plus élevée du jury, elle ne peut ordonner un renvoi qu’à l'unanimité de ses membres. Mais en permettant la division des questions sans préjudice pour l'accusé, le décret du 18 oct. 1848 sur la majorité du jury et plus encore la loi du 9 juin 1853 rendent les arguments de la Cour de cas- 
sation a priori sans effet bien que cette dernière campe sur ses positions.

(25) Gazette des tribunaux, 21 avr. 1827, aff. DUPRÉ.

(26) Parmi les 138 victimes de parricide dont nous connaissons la réaction (sur un total beaucoup plus large de 808 parents agressés ou tués), 62 prennent le parti de protéger l'enfant coupable.

(27) Code pénal, art. 309 et 312 .

(28) Code pénal, art. 312.

(29) Voir par exemple le Compte général de l'administration de la justice criminelle en France, année 1905, p. XIX.

(30) Code pénal, art. 319.

(31) AN, BB/20/251/ 1, aff. MIÉDAN, HauteMarne, 1863.

(32) AN, BB/20/208/ 1, aff. ENCLOS, Vosges, 1858.

(33) AN, BB/24/2058. DOS. 8962/S84, Joseph
Tandis que le parricide s'avère difficile à saisir aussi bien dans sa réalité matérielle que dans ses contours juridiques, l'attention du corps judiciaire tend en fait à se porter non plus exclusivement sur le crime mais de plus en plus sur le criminel. Le malaise suscité par le crime n'est sans doute pas étranger à cette réorientation du regard : il semble moins difficile d'essayer de cerner les motivations d'un coupable que d'envisager l'acte criminel dans toutes ses implications comme le souligne bien en 1884 le président de la cour d'assises de l'Isère à propos d'un accusé reconnu coupable d'un double parricide assorti d'un fratricide : «En résumé, dans cette triste cause, si les crimes commis, envisagés d'une manière abstraite, peuvent mériter la mort, l'homme ne la mérite point. ${ }^{(33)}$ "

\section{Du crime au criminel : le parricide sur la sellette}

Parce qu'il a levé le tabou suprême, l'auteur de parricide cristallise sur sa personne les interrogations angoissées du corps judiciaire partagé entre la nécessité d'une répression dissuasive et la prise de conscience de la diversité sinon de la complexité des cheminements parricides, prise de conscience qui en ébranlant les certitudes sur le crime permet de considérer celui-ci avec plus de nuance.

\section{L'épreuve de la confrontation judiciaire}

La confrontation de l'accusé avec la dépouille de sa victime, qui se tient la plupart du temps sur les lieux mêmes du crime et peut aller jusqu'à l'accomplissement de l'autopsie du cadavre sous les yeux de l'auteur présumé du meurtre, est l'un des passages obligés de l'instruction.

Dans les affaires de parricide, cette étape de la procédure constitue un moment clé dans la mesure où symboliquement, elle marque le rétablissement par la justice, pour un instant et a posteriori, des liens du sang irrémédiablement brisés par l'acte criminel. En somme, au-delà de la vertu ordalique ${ }^{(34)}$ attribuée ordinairement à cette épreuve, l'idée s'impose qu'à cette occasion, les liens du sang ne sauraient mentir : plus que pour tout autre crime, la vérité est appelée à jaillir de cette confrontation entre le meurtrier et sa victime originellement liés par une étroite parenté. Dès lors, aux yeux de la justice, la crainte d'un accusé d'être mis 
en présence du corps du défunt signe sans équivoque la culpabilité. Mais alors que cette mise en scène s'inscrit dans la religion de l'aveu qui anime classiquement la justice, très rares sont en réalité les parricides qui "craquent" devant le cadavre de leur parent décédé et se mettent à pleurer comme il est pourtant attendu d'eux : tel président de cour d'assises reproche à un fils parricide de n'avoir pas "essayé de pleurer" en face du cadavre de son père, tel autre fait grief à un fils meurtrier de sa mère de n'avoir pas même fait le "simulacre de se mouiller les yeux ${ }^{(36)}$ ". Les gens de justice voient évidemment dans cette impassibilité, très fréquente chez les parricides, matière à scandale, l'illustration sinon la preuve tangible de l'insensibilité du parricide. ${ }^{(37)}$ Cette attente immuable de la justice a toutefois quelque chose de spécieux puisque les éventuels pleurs d'un accusé, même survenus dès après le meurtre, se trouvent d'emblée suspectés et presque systématiquement taxés d'hypocrisie : "On peut se demander si ce n'était pas là des larmes de crocodile plutôt que des larmes sincères ${ }^{(38)}$ ", remarque à l'audience le président de la Cour à propos des pleurs versés par un fils à l'annonce de la disparition de son père. Pour la justice, de toute manière, la douleur ne peut avoir de sens que si elle manifeste l'existence de remords ${ }^{(39)}$ comme en témoignent en 1894 ces reproches adressés à un accusé par le président des assises de la Seine : "Vous faites celui qui pleure, mais vous ne pleurez pas sérieusement de vraies larmes de repentir. Ce sont celles là que je voudrais pouvoir vous arracher, et aussi un regret public pour un crime aussi abominable. Voyons, dites quelque chose à messieurs les jurés. ${ }^{(40)} "$

Dans le même ordre d'idée, les magistrats se montrent choqués sinon exaspérés de voir des accusés reconnaître parfois spontanément le meurtre d'un père ou d'une mère tout en refusant obstinément de céder sur des détails - l'aveu d'une conduite licencieuse par exemple ou de vols antérieurs au crime. Des monstres froids, ${ }^{(41)}$ c'est bien ainsi qu'apparaissent les parricides aux yeux des hommes de loi convaincus malgré les statistiques de la dangereuse progression du crime et partisans d'une répression plus sévère tant il est vrai que les parricides ne sont pas toujours jugés avec toute la rigueur énoncée dans le Code pénal.
JACQUIN, 1884. Nous soulignons.

(34) Mais contraire-

ment à l'ordalie et en particulier à la "cruentation”, phénomène qui renvoyait jusqu'à

l'époque moderne à

cette conviction que les plaies d'une victime d'assassinat étaient censées se rouvrir en présence du coupable, l'attention se porte ici sur les réactions de l'inculpé soumises au regard inquisiteur des protagonistes de l'instruction judiciaire.

(35) Gazette des tribunaux, 19 mai 1890, aff. ENJALBERT.

(36) Gazette des tribunaux, 7 nov. 1879, aff. DOYAT.

(37) Cette apparente indifférence renvoie semble-t-il davantage à l'effet de sidération consécutif à un traumatisme et largement attesté aujourd'hui par les psychiatres et criminologues. 
(38) Gazette des tribunaux, 19 juin 1898, aff. RAGUENEAU.

(39) Frédéric CHAUVAUD, "La parole captive. L'interrogatoire judiciaire au XIXème siècle", Histoire et archives, $\mathrm{n}^{\circ} 1$, janv.-juin 1997, p. 57.

(40) Gazette des tribunaux, 28 sept. 1894, aff. BOULAY.

(41) «Vous êtes froids, froids comme le morceau de bois que je tiens dans mes mains " lance aux accusés un président de cour d'assises en montrant une des pièces à conviction (Gazette des tribunaux, 4 déc. 1835, aff. RIZOU). Sur ce point, voir Frédéric CHAUVAUD, Les Criminels $d u$ Poitou au XIXème siècle, La Crèche, Geste éditions, 1999, pp. 116-126.

(42) Entre 1845 et 1853, 157 affaires de parricide sont jugées aux assises soit en moyenne un peu plus de 17 affaires par an alors

\section{Du parricide monstrueux au parricide tolérable}

C'est au milieu du siècle que la répression du parricide est la plus forte, au moment même où le crime est le plus fréquent, ${ }^{(42)}$ alors que le régime monarchique mais aussi le souvenir de la mise à mort royale de 1793 pèsent encore de tout leur poids. Dans les prétoires s'esquisse pourtant peu à peu une distinction entre le crime monstrueux et le crime tolérable. ${ }^{(4)}$

Si jusqu'en 1832, plus de la moitié des accusés de parricide sont acquittés, ${ }^{(44)}$ l'introduction généralisée des circonstances atténuantes après cette date, malgré quelques vives réticences, ${ }^{(4)}$ offre la possibilité de reconnaitre des degrés d'atrocité pour un crime a priori jugé sans excuse : "Il y a toujours quelque chose d'étrange, de voir admettre des circonstances atténuantes dans les accusations de parricide. Il faut néanmoins reconnaître que si ce crime n'est jamais excusable, il peut néanmoins être atténué par les circonstances dans lesquelles il a été commis. ${ }^{(46)}$ "

Sous l'action en particulier des avocats de la défense, ${ }^{(47)}$ juges et jurés en arrivent à examiner avec plus de souplesse le sens de la transgression consommée par le parricide tandis que les diagnostics en demi-teinte posés par les médecins lors des examens mentaux, qui distillent le doute quant à la santé morale des accusés sans pour autant les déclarer irresponsables, vont également dans le sens d'une modulation des peines. Dans la logique qui est la leur de décomposer le crime en ses différentes composantes, les magistrats réintroduisent dans les questions soumises au jury les notions de guet-apens et surtout de préméditation ${ }^{(48)}$ pourtant sans valeur légale dans le cas du parricide, crime constitué indépendamment de toute circonstance : "Pour des criminalistes, la mort $d u$ père par le fils est placée sur le même degré de l'échelle pénale que l'assassinat mais pour des jurés qui jugent humainement, la préméditation ainsi que le guet-apens indiquent seuls que le meurtrier a atteint le dernier degré de la perversité. A celui-là seul ils réservent le dernier supplice. ${ }^{(49)} "$

Il n'en demeure pas moins que les magistrats ne parviennent guère à distinguer ce qui, au sens précis du terme juridique, peut relever de la préméditation et ce qui ressortit davantage à une froide détermination 
liée à une escalade incontrôlée de la violence dans le cadre d'un quotidien partagé.

Aux deux extrémités de l'échelle pénale se dessine alors une évolution majeure, la diminution du nombre des acquittements accompagnant un évitement croissant de la peine de mort. Témoignant de l'écart entre la norme et la pratique puisque $25 \%$ des accusés de parricide sont encore acquittés au début du XXème siècle ${ }^{(50)}$ le traitement judiciaire des affaires de parricide renvoie à l'élaboration dans les prétoires d'une perception plus nuancée du crime qui tend ainsi à rééquilibrer en faveur des enfants la dissymétrie répressive édictée par le Code pénal. En prenant le parti de ne plus porter son attention uniquement sur le crime mais aussi sur les criminels, la justice individualise le crime pour mieux tenir compte du contexte familial qui a rendu l'homicide possible ; du même coup, elle s'intéresse aux victimes dont le statut en arrive à être remis en cause. Derrière l'image du père défaillant, de plus en plus brandie dans la seconde moitié du siècle, se profile dès lors celle autrement insoutenable du père coupable voire criminel. En 1907 par exemple, pourtant reconnu responsable du meurtre de son père, Émile C., honnête et laborieux manœuvre de 27 ans, n'écope que de cinq ans de travaux forcés, par la suite commués en cinq ans de prison : c'est que le père, jouissant d'une réputation détestable, avait rendu la vie insupportable aux siens en faisant d'eux de véritables martyrs. ${ }^{(51)}$

Tandis que certains parricides adultes commencent à profiter d'une relative indulgence dès le début des années 1860, l'affinement du jugement porté sur les jeunes parricides, plus étroitement soumis à l'émergence effective de l'enfant comme sujet de droit, ${ }^{(52)}$ n'intervient que trente ans plus tard, preuve s'il en est du caractère sacro-saint de la puissance paternelle dans la France du XIXème siècle.

\section{Le supplice des parricides}

Si la peine capitale tend à être prononcée avec plus de parcimonie au fil du siècle - elle concerne environ $30 \%$ des condamnés au début du siècle mais plus que $15 \%$ à partir des années $1890^{(53)}$-, l'article 13 du Code pénal, spécificité française, ${ }^{(54)}$ entoure le supplice des parricides d'un appa- que la moyenne annuelle pour le siècle est de 12 .

(43) Sylvie LAPALUS, "Le parricide comme exacerbation...”, art. cit., p. 145.

(44) Pourcentage établi à partir du Compte général.

(45) «Il serait à désirer que le parricide, que le Code pénal ne considère pas comme excusable, ne fut jamais atténué par des circonstances qui n'existent que bien rarement et qui presque toujours sont créées arbitrairement par le jury.

Dans des cas extraordinaires, le Roi pourrait modifier la peine, et tout serait alors concilié » (AN, BB/20/133, aff. DELAVIGNE, Côtes-du-Nord, 1845 , résumé du président des assises).

(46) AN, BB/20/263/ 2, aff. LAURENT, Loire, 1864, résumé du président de la cour d'assises.

(47) Dans les affaires de parricide, la défense est souvent confiée à des avocats inexpérimentés, 
sans doute parce que la réprobation générale qui s'attache au parricide rend la tâche délicate et n'en fait pas de belles causes à défendre.

(48) Raoul LAJOYE (avocat à la cour d'appel), De la préméditation dans le parricide et dans l'infanticide, Paris, A. Parent, 1875, 22 p.

(49) AN, BB/20/172/ 1, aff. GLANDUS, Haute-Vienne, 1854, résumé du président de la cour d'assises. Nous soulignons.

(50) Pourcentage établi à partir des données du Compte général.

(51) AD Saône-etLoire, U 1053, aff. Émile C., 1907.

(52) Lois du 24 juil. 1889 et du 19 avr. 1898. Voir par exemple Bernard SCHNAPPER, "Un nouveau regard sur l'enfance", in Enfants corrigés, enfants protégés. Genèse de la protection de l'enfance en Belgique, en France, aux Pays-Bas et au reil particulier : le condamné est en effet conduit sur le lieu de l'exécution en chemise, nu-pieds et la tête couverte d'un voile noir avant d'être exposé sur l'échafaud pendant la lecture par l'huissier de l'arrêt de condamnation. Et, jusqu'à la loi du 28 avril 1832 qui, en supprimant certaines rigueurs du Code, marque une nouvelle étape dans le processus de désincarnation du droit pénal, ${ }^{(55)}$ le parricide subit aussi l'amputation de la main droite. ${ }^{(56)}$ Cette aggravation de la peine s'inscrit dans la continuité des siècles précédents qui réservaient toujours au parricide un châtiment spécial.

Une fois promis à un tel supplice, le condamné devient en prison l'objet d'un traitement spécial, un prisonnier dont on surveille tous les faits et gestes - son appétit, son humeur, son sommeil et jusqu'aux rêves de ses nuits de captivité -, un prisonnier à qui l'administration pénitentiaire octroie souvent un compagnon de cellule tenant lieu de mouchard et devant empêcher toute tentative de suicide : un prisonnier dont la société attend jusqu'au dernier instant non seulement l'aveu de l'acte criminel, assorti si possible de l'expression sincère du remords, mais surtout la vérité ultime sur le crime. A travers l'exécution du parricide, les magistrats veulent donner à voir le caractère inéluctable de la punition et la force d'un châtiment exemplaire, ce que l'historiographie a bien mis en valeur ${ }^{(57)}$ : "Ce qu'il nous importait d'imprimer au sein des populations, c'est cette vérité terrible et salutaire que les crimes les plus secrets sont découverts tôt ou tard et souvent par le côté qu'on a le moins soupçonné. Le châtiment arrive quelquefois lentement, mais il arrive avec certitude. ${ }^{(58)}$ »

De ce fait, le corps judiciaire se montre très soucieux de l'endroit où seront dressés les bois de la guillotine - au plus près des populations, sur les lieux mêmes du crime le plus souvent -, surtout dans les contrées que les magistrats, incarnant les valeurs de la civilisation, s'accordent à considérer comme sauvages, l'idée étant que la morale du peuple se règle sur les décisions de justice.

En tout cas, présidents des assises et procureurs généraux voient d'un très mauvais œil les commutations de peine accordées aux condamnés à mort pour parricide dans la mesure où ces dernières sont susceptibles d'avoir un effet fâcheux sur l'opinion publique en accréditant l'opinion 
qu'à défaut d'être supprimée, la peine capitale serait à tout le moins tombée en désuétude : si la peine de mort ne s'applique même plus au parricide, c'est à n'en pas douter la porte ouverte à tous les crimes ! Après la révolution de 1848 en particulier, dans le cadre de l'offensive morale menée par le parti de l'Ordre, les magistrats désapprouvent plus que jamais les commutations ou remises de peine qui risquent selon eux d'être interprétées au sein de la population comme un acte de faiblesse de la part du gouvernement et pourraient même inciter à croire que l'avènement de la République signifie l'abolition totale de la peine de mort, ${ }^{(59)}$ proclamée uniquement en matière politique le 26 février 1848 : en ces temps de démoralisation supposée de la population, il importe de réaffirmer plus que jamais la force du lien familial que la magistrature croit menacé par la diffusion des idées socialistes. Jusque dans les années 1880 , les condamnés à mort pour parricide voient leur peine commuée dans moins de $10 \%$ des cas, ${ }^{(60)}$ proportion qui passe à plus de $62 \%$ après cette date, ${ }^{(61)}$ dans un contexte moins répressif à l'encontre des parricides mais aussi dans celui d'une contestation plus forte de la peine de mort et alors que l'assimilation entre parricide et régicide perd malgré tout de son actualité : vers la fin du siècle, si la proclamation de la peine capitale semble encore nécessaire à plus d'un magistrat, son exécution semble en revanche moins indispensable, même en cas de parricide. ${ }^{(62)}$

En déplaçant progressivement son regard du crime sur le criminel, la justice du XIXème siècle parvient à envisager avec moins d'effroi un crime qui, abordé frontalement, la laisse sans prise. Mais ce déplacement de perspective n'est pas sans conséquence puisqu'il se fait au prix d'un éventuel amoindrissement de la responsabilité du criminel. Sous l'influence de l'école criminologique française notamment, la vision organiciste du parricide inspirée en partie des principes du criminel-né de Cesare Lombroso s'efface subrepticement devant le sentiment que la famille peut elle-même enfanter le criminel et cette prise de conscience lézarde encore plus sûrement l'édifice social que le crime en lui-même : "On ne pouvait se défendre de la pensée que ce malheureux, placé dans un autre
Québec (1820-1914),

Rapport de recherche, ministère de la Justice, Paris, 1995, pp. 177-184.

(53) Pourcentages établis à partir du Compte général.

(53) Édouard FUZIER-HERMAN, Répertoire général alphabétique du droit français, Paris, 1886-1906, t. 29, art "Parricide", chap. IV (Droit comparé).

(55) Jean-Pierre BAUD, L'Affaire de la main volée. Une histoire juridique du corps, Paris, Seuil, 1993, p. 105.

(56) Au sein de notre corpus, sur 13 parricides condamnés au supplice entre 1825 et 1832 , seuls 3 se voient gratifiés de la remise du poing au moment de l'exécution, et ce en 1831 ou 1832 donc en anticipation de la loi du 28 avr. 1832.

(57) On citera évidemment Michel FOUCAULT, Surveiller et Punir, Paris, Gallimard, 1993, 363 p. ; Pieter 
SPIERENBURG, The

Spectacle of suffering.

Executions and the evolu-

tion of repression : from a preindustrial metropolis to the European experience, Cambridge, Cambridge University Press, 1984, $274 \mathrm{p}$.

(58) AN, BB/20/135, aff. MONTAGNER, Haute-Vienne, 1846, rapport du président des assises.

(59) Par exemple :

AN, BB/20/146/1, aff.

PRINCE, Gironde, 1849.

(60) Soit 15 commutations de peine sur 162 demandes répertoriées par nous jusqu'en 1879 dans la série $\mathrm{BB} / 24$ des Archives nationales. (61) Soit 37 commutations accordées pour 59 demandes effectuées entre 1880 et 1913.

(62) AN, BB/24/2092. DOS. 1411/S05, Achille L., 1905, avis du président des assises sur une éventuelle commutation de peine. milieu, et avec un autre père, aurait pu devenir un honnête homme ${ }^{(63)}$ " conclut un président de cour d'assises à propos d'un jeune homme de 21 ans meurtrier de son père en $1858 \ldots$ 